\title{
Guest Editorial: Smart Multimedia Communication Technologies for New-Generation Artificial Intelligence Applications
}

\author{
Wen-Hsiang Hsieh ${ }^{1}$
}

Published online: 5 August 2016

(C) Springer Science+Business Media New York 2016

\section{Introduction}

With the development of the Internet of thing (IoT) mechanism nowadays, the required techniques for artificial intelligence (AI)-related applications will be equipped with a crucial property, which is "smart." Smart multimedia communication technologies are composed of two main technique categories: the smart multimedia processing technique class and the smart communication transmission technique class. These will be the necessity for constructing a new-generation AI application. The technical category of multimedia processing involves lots of types of techniques where pattern recognition, video (or image) processing, and human machine interaction (HCI) are three kernel technique types to establish an AI application under the regulation of the IoT mechanism. Video coding and decoding (codecs) techniques, media streaming techniques, and hardware-based techniques for enhancing media communication play important roles for establishing an efficient communication transmission channel between the user end device and the remote media handling server.

"Smart multimedia communication technologies" associated with the new-generation AI application will absolutely be an evolution tendency for the development of multimedia and communication technologies. Following this thought line, we deeply believe that the appropriate term "smart" will be given to pattern recognition, video processing, human machine interaction, media streaming, and hardware-based media communication enhancement techniques for building up a smart multimedia communication system.

The special issue, "Smart Multimedia Communication Technologies for New-Generation Artificial Intelligence Applications," acts as a path breaker for exploring new-generation AI applications using multimedia communication technologies with intelligence. We totally received around 30 submissions, and the vast majority of all submissions are significant in

Wen-Hsiang Hsieh

allen@nfu.edu.tw

1 Department of Automation Engineering, National Formosa University, Huwei Township, Yunlin, Taiwan 
the fields of multimedia processing and communication transmission. After an extremely strict review process, only 22 papers with high quality were accepted. The accepted papers are within the technical scope of this special issue and belong to one of "smart pattern recognition," "smart video processing," "smart human machine interaction," "smart media streaming," and "smart hardware-based media communication enhancement" category techniques.

\section{Summary of 22 accepted papers}

The first seven papers belong to the technique category of smart pattern recognition $[4,7,10$, 13, 14, 18, 22]. The first paper entitled "Feature design scheme for Kinect-based DTW human gesture recognition" by Ding and Chang [7] develops a feature design scheme involving hybridizations of joint positions and joint angles for dynamic time warping-based human gesture recognition with the Kinect camera. The second paper entitled "Implementation of an interactive TV interface via gesture and handwritten numeral recognition" by Sheu and Huang [14] considers the utilization of the Kinect sensor to recognize the dynamic gestures of users to achieve real-time interactive control of TV where a back-propagation neural network was also used to perform handwriting recognition to help the command recognition task. The third paper entitled "An novel random forests and its application to the classification of mangroves remote sensing image" by Luo et al. [13] proposes a novel random forests algorithm with variables random input and random combination machine to improve the weakness of low accuracy and an over-fitting phenomenon in a single decision tree. The fourth paper entitled "Dynamic image segmentation algorithm in 3D descriptions of remote sensing images" by Chen et al. [4] addresses a technique issue in 3D descriptions of remote sensing images where a dynamic image segmentation algorithm is presented to result in the new boundary from image contents. The fifth paper entitled "Frequency domain digital watermark recognition using image code sequences with a back-propagation neural network" by Yen and Huang [22] explores developments of a digital watermarking scheme by combining the DCT and BPNN methods. The sixth paper entitled "Automatic footprint detection approach for the calculation of arch index and plantar pressure in a flat rubber pad" by Su et al. [18] focuses on the technique issue of the calculation of arch index and plantar pressure in a flat rubber pad where an automatic footprint detection approach by employing Otsu's threshold method is proposed. The seventh paper entitled "A gender classification scheme based on multi-region feature extraction and information fusion for unconstrained images" by Lin et al. [10] considers the issue on multi-region feature extraction and information fusion for gender recognition where pre-processing, multi-region feature extraction, and gender classifier are separately designed.

Among all the accepted papers, there are four papers in the smart media streaming category. The work done by Sheu et al. [15] concentrates on the issue of audio streaming where a smart Ethernet-based synchronous audio playback system is realized. The investigation carried out by Chen et al. [2] addresses the issue concerning the deployment of a radio monitoring system using software-defined radio technologies where the proposed smart architecture is a cost-effective and flexible platform as compared with traditional spectrum monitoring systems. The tenth and the 11th papers by Lin et al. [1, 12] focus on the promising issue of advanced video encoding and decoding (advanced video coding, AVC) for smart video-data streaming. A prediction technique for the best coding unit partition tree is proposed for high-efficiency video coding in [12]; a dynamic reconfigurable coding-decoding scheme based on a chaotic sequence is developed to enhance the security of H.264/AVC streaming in [1]. 
The technology category of smart video processing contains four papers $[5,8,11,20]$. The 12th paper presented by Hsia et al. [8] discusses the promising issue of developments of an efficient video retrieval technique for smart video synopsis. The 13th paper presented by Lin and $\mathrm{Ji}$ [11] indicates an issue to concern the utilization of visual attention model and multilevel exposure correction on video quality enhancement. The 14th paper by Wang et al. [20] points out the effect of the loss of packet on visual quality. The authors propose an efficient full frame algorithm for depth-based 3D videos. The 15th paper by Chen et al. [5] focuses on the image processing issue. The authors propose a particle swarm optimization approach to automatically evolve the multiplier-less 2D SMDWT filter hardware architecture for implementing a high-efficiency embedded image-processing system with smart.

There are seven papers $[6,9,16,17,19]$ in the technology category of smart human machine interaction. These studies focus on the technique issue to realize a human computer interaction system for the specific application. Remote e-teaching systems for students $[6,9,16]$ in the field of education and remote rehabilitation systems for patients in the field of home care [17] are two main smart human interaction interface applications. The 20th paper presented by Ueng and Chen [19] proposes a vision-based multi-user human computer interaction method for creating augmented reality user interfaces. The presented system in [19] is a smart HCI system, efficient, flexible, and practical for users with problems on using ordinary input devices.

The final two papers are in the smart hardware-based media communication category [3, 21]. The study presented by Wang et al. in [21] discusses the promising issue relating to digital forensic data from volatile system memory. A computer forensic analysis model for the reconstruction of a chain of evidence of volatile memory data is proposed. The work done by Chen et al. in [3] then considers the issue on the use of geometry image for GPUaccelerated blind and robust 3D mesh watermarking. The study points out that the embedding and detection process is extremely fast with the help of parallel computations on the GPUs.

\section{Conclusions}

All of the innovative ideas, concepts, and designs in smart multimedia communication technologies will have the considerable benefit to prompt researchers engaged in related work or readers that are interested in the technique topic to obtain state-of-the-art knowledge. We thank the authors who submitted their work and the technical reviewers who were a great help for strictly considering the technique correctness and the research quality.

Acknowledgments I would like to express my sincere appreciation to all the authors who have submitted their work for publication in this Special Issue. I am also grateful to the many reviewers that provided their time and expertise in conducting valuable peer reviews. In addition, I deeply thank all the staffs of the Editorial Department of the journal for their kind help with the processing of the publication of this issue. Finally, special thanks must be given to the Editor in Chief Prof. Borko Furht for his kind support to the publication of this issue.

\section{References}

1. Chang Y-T, Lin Y-C (2015) Dynamic reconfigurable encryption and decryption with chaos/M-sequence mapping algorithm for secure H.264/AVC video streaming over OCDMA passive optical network. Multimed Tools Appl. doi:10.1007/s11042-015-2784-1

2. Chen W-T, Chang K-T, Ko C-P (2015) Spectrum monitoring for wireless TV and FM broadcast using software-defined radio. Multimed Tools Appl. doi:10.1007/s11042-015-2764-5 
3. Chen H-K, Chen, W-S (2015) GPU-accelerated blind and robust 3D mesh watermarking by geometry image. Multimed Tools Appl. doi:10.1007/s11042-015-3062-y

4. Chen C-Y, Feng H-M, Chen H-C, Jou S-M (2015) Dynamic image segmentation algorithm in 3D descriptions of remote sensing images. Multimed Tools Appl. doi:10.1007/s11042-015-2795-y

5. Chen C-Y, Hsia C-H, Yang C-Y (2015) Evolutionary design of multiplierless lifting-based 2D DWT filters for low-resolution image processing. Multimed Tools Appl. doi:10.1007/s11042-015-2776-1

6. ChiangK-H, Fan C-Y, Liu H-H, Chen, G-D (2015) Effects of a computer-assisted argument map learning strategy on sixth-grade students' argumentative essay reading comprehension. Multimed Tools Appl. doi:10. 1007/s11042-015-2904-y

7. Ding I-J, Chang C-W (2015) Feature design scheme for Kinect-based DTW human gesture recognition. Multimed Tools Appl. doi:10.1007/s11042-015-2782-3

8. Hsia C-H, Chiang J-S, Hsieh C-F (2015) Low-complexity range tree for video synopsis system. Multimed Tools Appl. doi:10.1007/s11042-015-2714-2

9. Ko H-C (2015) An approach to the design of digital classic Chinese article learning system for undergraduate students using "The Homecoming" as an example. Multimed Tools Appl. doi:10.1007/s11042-015-3126-Z

10. Lin G-S, Chang M-K, Chang Y-J, Yeh C-H (2015) A gender classification scheme based on multi-region feature extraction and information fusion for unconstrained images. Multimed Tools Appl. doi:10.1007/ s11042-015-2797-9

11. Lin G-S, Ji X-W (2015) Video quality enhancement based on visual attention model and multi-level exposure correction. Multimed Tools Appl. doi:10.1007/s11042-015-2777-0

12. Lin Y-C, Lai J-C, Cheng H-C (2015) Coding unit partition prediction technique for fast video encoding in HEVC. Multimed Tools Appl. doi:10.1007/s11042-015-2778-Z

13. Luo Y-M, Huang D-T, Liu P-Z, Feng H-M (2015) A novel random forests and its application to the classification of mangroves remote sensing image. Multimed Tools Appl. doi:10.1007/s11042-015-2906-9

14. Sheu J-S, Huang Y-L (2015) Implementation of an interactive TV interface via gesture and handwritten numeral recognition. Multimed Tools Appl. doi:10.1007/s11042-015-2739-6

15. Sheu J-S, Shou H-N, Lin W-J (2015) Realization of an Ethernet-based synchronous audio playback system. Multimed Tools Appl. doi:10.1007/s11042-015-2983-9

16. Su C-H (2015) The effects of students' motivation, cognitive load and learning anxiety in gamification software engineering education: a structural equation modeling study. Multimed Tools Appl. doi:10.1007/ s11042-015-2799-7

17. Su C-H (2015) Developing and evaluating effectiveness of 3D game-based rehabilitation system for total knee replacement rehabilitation patients. Multimed Tools Appl. doi:10.1007/s11042-015-2820-1

18. Su K-H, Kaewwichit T, Tseng C-H, Chang C-C (2015) Automatic footprint detection approach for the calculation of arch index and plantar pressure in a flat rubber pad. Multimed Tools Appl. doi:10.1007/ s11042-015-2796-X

19. Ueng S-K, Chen G-Z (2015) Vision based multi-user human computer interaction. Multimed Tools Appl. doi:10.1007/s11042-015-3061-Z

20. Wang C-C, Hong C-S, Tai S-C, LuoY-C (2015) An efficient full frame algorithm for object-based error concealment in 3D depth-based video. Multimed Tools Appl. doi:10.1007/s11042-015-2899-4

21. Wang F, Hu L, Hu J, Zhao K (2015) Computer forensic analysis model for the reconstruction of chain of evidence of volatile memory data. Multimed Tools Appl. doi:10.1007/s11042-015-2798-8

22. Yen C-T, Huang Y-J (2015) Frequency domain digital watermark recognition using image code sequences with a back-propagation neural network. Multimed Tools Appl. doi:10.1007/s11042-015-2718-y 